\title{
Control and stability of the lateral dynamics of the vehicle to avoid road accidents
}

\author{
Daniel Sava ${ }^{1}$ and Ion Copae ${ }^{2, *}$ \\ ${ }^{1}$ S.A-R City Insurance, 12-14 Father Galeriu Str., Bucharest, Romania. \\ 2 Department of Military Vehicles and Transportation, Military Technical Academy "Ferdinand I", 39-49 George Cosbuc \\ Blvd., Bucharest, Romania.
}

Global Journal of Engineering and Technology Advances, 2021, 06(02), 159-168

Publication history: Received on 20 January 2021; revised on 22 February 2021; accepted on 24 February 2021

Article DOI: https://doi.org/10.30574/gjeta.2021.6.2.0029

\begin{abstract}
The paper presents elements related to the control and stability of the lateral dynamics of the vehicle. The mathematical model of the ESC system is given. The study of stability is presented, including by using the phase portrait. The control strategies and algorithms used in ESC are presented. The results obtained using the technical data of the Volkswagen Touareg and Chevrolet Camaro cars are presented.
\end{abstract}

Keywords: Electronic stability control; ESC; ESP; Phase portrait; Lateral dynamics

\section{Introduction}

Current vehicles are equipped with active stability control systems, which ensure the prevention of leaving the desired trajectories of movement, horizontally and vertically; for this purpose, the control of the yaw and rolling movements is ensured, separately or through an integrated solution $[1,2,3]$. Current solutions also ensure the control of longitudinal dynamics, for example with the help of the adaptive cruise control system (ACC: Adaptive Cruise Control) and the antilock braking system (ABS: Anti-lock Brake System). The purpose of this paper is to study the control of the lateral dynamics of the vehicle using the electronic stability control system (ESC: Electronic Stability Control; ESP: Electronic Stability Program).

\section{Mathematical model}

In the literature, the most used mathematical model of the lateral dynamics of the vehicle is the one with two degrees of freedom [4]. The two degrees of freedom of the model are the angular speed of yaw movement $\omega_{z}$ (yaw rate) and the angle of lateral sliding $\beta$.

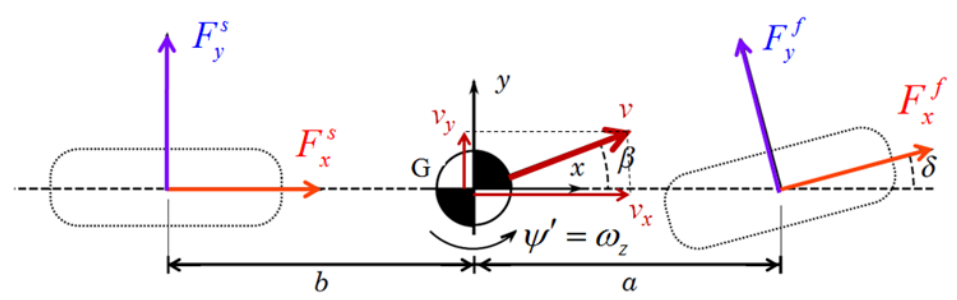

Figure 1 The two-degree freedom model of lateral dynamics

\footnotetext{
* Corresponding author: Ion Copae; Email: copaeion@yahoo.com

Military Technical Academy "Ferdinand I", Bucharest, Romania.

Copyright $(2021$ Author(s) retain the copyright of this article. This article is published under the terms of the Creative Commons Attribution Liscense 4.0.
} 
Fig. 1 shows the calculation scheme of this mathematical model, which shows the lateral forces $F_{y}^{f}$ and $F_{y}^{s}$, the first at the front axle, the second at the rear axle; longitudinal forces at the axles are also presented, $F_{x}^{f}$ and $F_{x}^{s}$.

The lateral sliding angle of the vehicle is determined by the relation, with $v_{y}$ and $v_{x}$ the axial components of the velocity $v$ :

$$
\beta=\operatorname{arctg} \frac{v_{y}}{v_{x}}
$$

Also in fig. 1 are shown the steering angle of the front wheels $\delta$, as well as the distances $a$ and $b$ from the center of gravity $\mathrm{G}$ to the axles.

The mathematical description of the mathematical model in fig.1 is:

$$
\left\{\begin{array}{l}
m v_{x}\left(\beta^{\prime}+\omega_{z}\right)=F_{y}^{f} \cos \delta+F_{y}^{s}+F_{x}^{s} \sin \delta \\
J_{z} \omega_{z}^{\prime}=a F_{y}^{f} \cos \delta-b F_{y}^{s}+a F_{x}^{f} \sin \delta
\end{array}\right.
$$

\section{Stability of lateral movement}

Due to the presence of trigonometric functions in expressions (2), the mathematical model is nonlinear, which is a difficulty in controlling stability using ESP. For this reason, the model is usually linearized taking into account that in practice the angle values $\delta$ are small at each time/calculation step and consequently the system (2) becomes:

$\left\{\begin{array}{l}m v_{x}\left(\beta^{\prime}+\omega_{z}\right)=F_{y}^{f}+F_{y}^{s} \\ J_{z} \omega_{z}^{\prime}=a F_{y}^{f}-b F_{y}^{s}\end{array}\right.$

In the frequently adopted hypothesis of a linear model of the tire, the relations result:

$$
F_{y}^{f}=C_{\alpha}^{f} \alpha_{f} ; F_{y}^{s}=C_{\alpha}^{s} \alpha_{s}
$$

in which $C_{\alpha}^{f}$ and $C_{\alpha}^{s}$ represents the transverse stiffness of the front and rear axle tires, respectively; in addition, $\alpha_{f}$ and $\alpha_{s}$ represent the lateral sliding angle of the front wheel and the rear wheel, respectively, established with the relations (the steering is only at the front axle):

$$
\alpha_{f}=\delta-\frac{v_{x} \beta+a \omega_{z}}{v_{x}} ; \alpha_{s}=-\frac{v_{x} \beta-b \omega_{z}}{v_{x}}
$$

The system of differential equations is finally obtained:

$$
\left\{\begin{array}{l}
m v_{x}^{2} \beta^{\prime}+\left(C_{\alpha}^{f} v_{x}+C_{\alpha}^{s} v_{x}\right) \beta+\left(m v_{x}^{2}+a C_{\alpha}^{f}-b C_{\alpha}^{s}\right) \omega_{z}=C_{\alpha}^{f} v_{x} \delta \\
\left(a C_{\alpha}^{f} v_{x}-b C_{\alpha}^{s} v_{x}\right) \beta+J_{z} v_{x} \omega_{z}^{\prime}+\left(a^{2} C_{\alpha}^{f}+b^{2} C_{\alpha}^{s}\right) \omega_{z}=a C_{\alpha}^{f} v_{x} \delta
\end{array}\right.
$$

Applying the Laplace transform to the relations (6) we obtain the main determinant: 
$\Delta=\left|\begin{array}{cc}m v_{x}^{2} s+C_{\alpha}^{f} v_{x}+C_{\alpha}^{s} v_{x} & m v_{x}^{2}+a C_{\alpha}^{f}-b C_{\alpha}^{s} \\ a C_{\alpha}^{f} v_{x}-b C_{\alpha}^{s} v_{x} & J_{z} v_{x} s+a^{2} C_{\alpha}^{f}+b^{2} C_{\alpha}^{s}\end{array}\right|$

from which results the characteristic equation of the form:

$\Delta=0 \Rightarrow A(s)=a_{2} s^{2}+a_{1} s+a_{0}=0$

Where: $\left\{\begin{array}{l}a_{2}=J_{z} m v_{x}^{3} \\ a_{1}=\left(C_{\alpha}^{f} J_{z}+C_{\alpha}^{s} J_{z}+a^{2} C_{\alpha}^{f} m+b^{2} C_{\alpha}^{s} m\right) v_{x}^{2} \\ a_{0}=(a+b)^{2} v_{x} C_{\alpha}^{f} C_{\alpha}^{s}+\left(b C_{\alpha}^{s}-a C_{\alpha}^{f}\right) m v_{x}^{3}\end{array}\right.$

From systems theory it is known that a system is stable if the roots of the characteristic equation $A(s)=0$ are real and negative, or complex with the real negative part. For a second order system (as in this case), the necessary and sufficient condition for the system to be stable is that all the coefficients of the characteristic equation are strictly positive, in which case the roots satisfy the mentioned conditions. From expressions (9) it is found that $a_{2}>0$ and $a_{1}>0$. From the condition that $a_{0}>0$ results:

$$
(a+b)^{2} C_{\alpha}^{f} C_{\alpha}^{s}+\left(b C_{\alpha}^{s}-a C_{\alpha}^{f}\right) m v_{x}^{2}>0
$$

\section{Results and discussion}

Fig. 2 shows the lateral slip angle $\beta$ and the angular speed $\omega_{z}$ using the technical data of the Volkswagen Touareg car, for two values of the longitudinal speed $\left(V_{x}=80 \mathrm{~km} / \mathrm{h}\right.$ and $\left.V_{x}=110 \mathrm{~km} / \mathrm{h}\right)$ and for the angle front steering wheel $\delta=10$ degrees.
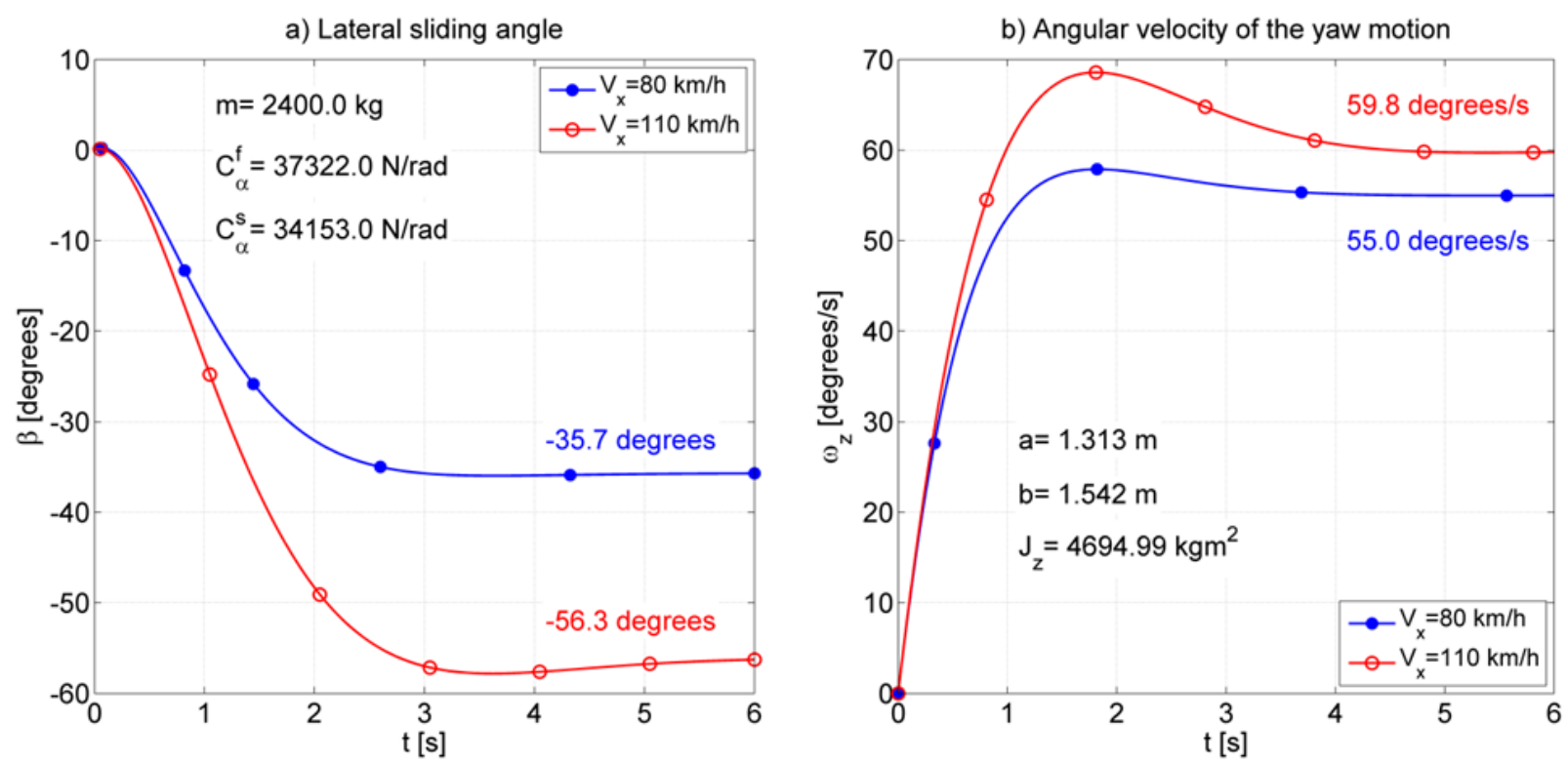

Figure 2 Lateral sliding angle and angular velocity of the yaw motion 
As can be seen from fig. 2, all curves tend towards a finite value, which is the stationary value, or the static equilibrium value; since the stationary value is finite, it follows that the stability of the stationary regimes is ensured and therefore the condition is fulfilled (10). It is therefore necessary to analyze the stability of stationary regimes for different values of longitudinal speed, wheel steering angle and tire side stiffness. For this purpose, the expressions (6) are known as:

$$
\left\{\begin{array}{l}
\beta^{\prime}=-\omega_{z}+c_{1}\left(\delta-\beta-c_{2} \omega_{z}\right)+c_{3}\left(-\beta+c_{4} \omega_{z}\right) \\
\omega_{z}^{\prime}=c_{5}\left(\delta-\beta-c_{2} \omega_{z}\right)-c_{6}\left(-\beta+c_{4} \omega_{z}\right)
\end{array}\right.
$$

where:

$c_{1}=\frac{C_{\alpha}^{f}}{m v_{x}} ; c_{2}=\frac{a}{v_{x}} ; c_{3}=\frac{C_{\alpha}^{s}}{m v_{x}} ; c_{4}=\frac{b}{v_{x}} ; c_{5}=\frac{a C_{\alpha}^{f}}{J_{z}} ; c_{6}=\frac{b C_{\alpha}^{s}}{J_{z}}$

In steady state, $\beta=$ const. and $\omega_{z}=$ const. and as a result in the left limb of equations (11) results $\beta^{\prime}=0$ and $\omega_{z}^{\prime}=0$. The expressions (11) thus become algebraic equations, and their solutions are stationary regime points (static equilibrium points):

$$
\left\{\begin{array}{l}
-\omega_{z}+c_{1}\left(\delta-\beta-c_{2} \omega_{z}\right)+c_{3}\left(-\beta+c_{4} \omega_{z}\right)=0 \\
c_{5}\left(\delta-\beta-c_{2} \omega_{z}\right)-c_{6}\left(-\beta+c_{4} \omega_{z}\right)=0
\end{array}\right.
$$

For example, in fig. 3, for the longitudinal speed $V_{x}=90 \mathrm{~km} / \mathrm{h}$ and the steering angle of the wheel $\delta=15$ degrees, the stationary point is $\mathrm{A}(86 ;-64.3)$.

a) Lateral sliding angle $\beta$

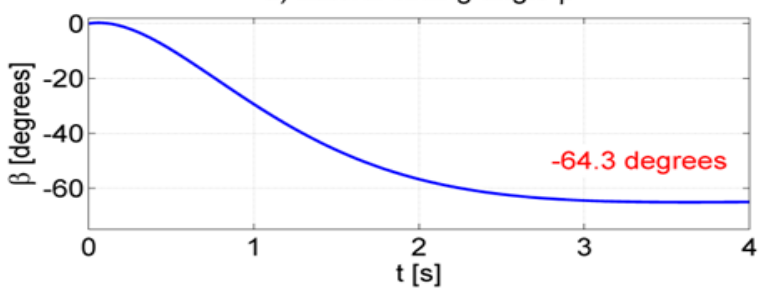

b) Angular velocity of the yaw motion $\omega_{z}$

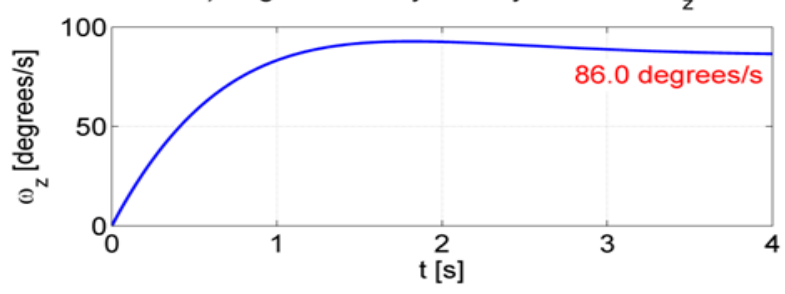

c) Static equilibrium $\beta=f\left(\omega_{z}\right)$

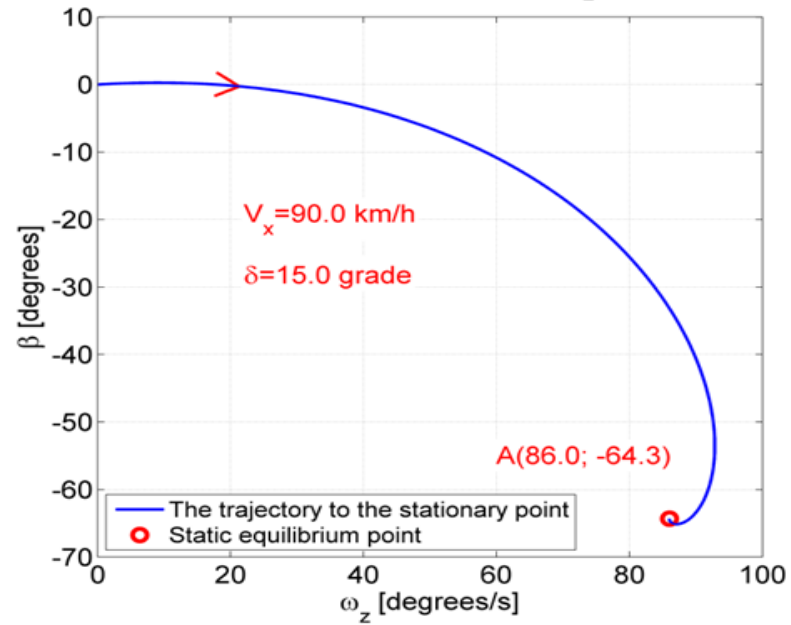

Figure 3 Establishing the stationary regime point

By proceeding similarly, the results of fig. $4 \mathrm{a}$ are obtained at different speeds $V_{x}$ for $\delta=10$ degrees and in fig. $4 \mathrm{~b}$ at different angles $\delta$ and at $V_{x}=80 \mathrm{~km} / \mathrm{h}$. The graphs in fig. 4 show that the stationary regimes are stable, each curve having a point towards which the values of the two quantities tend. 
a) For different $\mathrm{V}_{\mathrm{x}}$ and $\delta=10$ grade

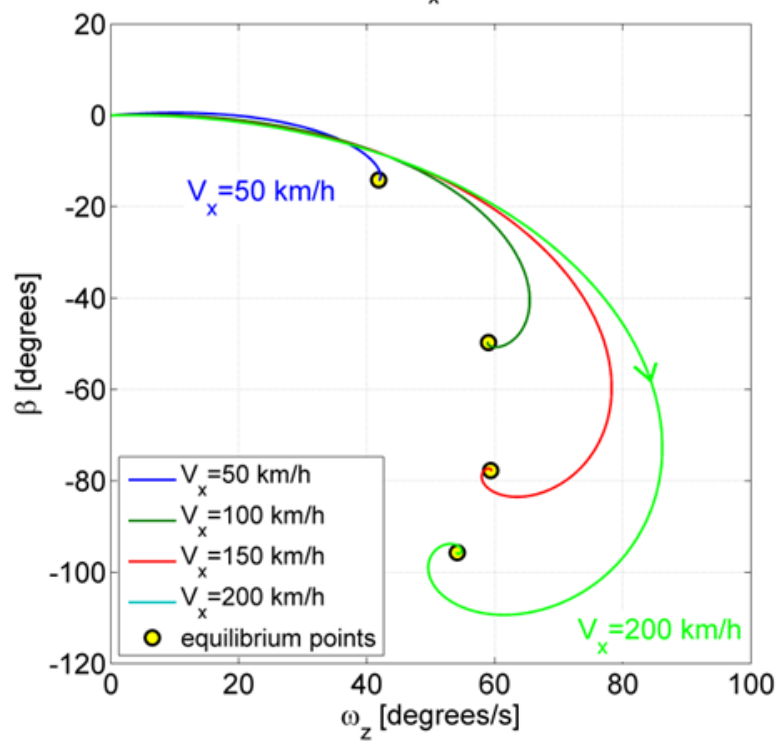

b) For different $\delta$ and $V_{x}=80 \mathrm{~km} / \mathrm{h}$

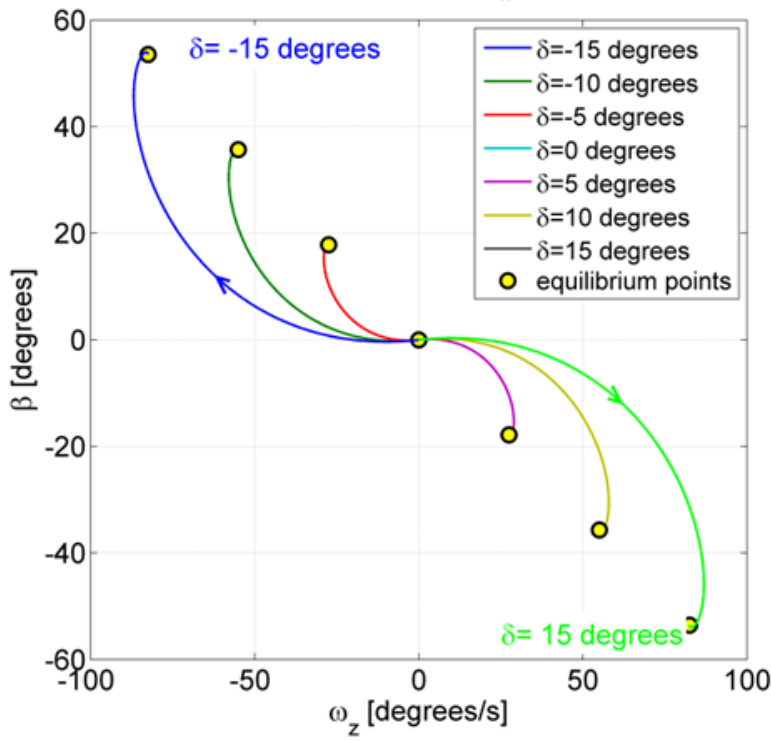

Figure 4 Study of the stability of stationary regimes

The stability of the system can also be studied based on the phase portrait, which contains the vector field, the phase trajectories, the critical points and possibly the isoclines. If this portrait is rendered in the plan, then there is the phase plan, and if it is illustrated in space, then there is the phase space. For the case in question above, it follows from relations (13):

$\left\{\begin{array}{l}\beta\left(-c_{1}-c_{3}\right)+\omega_{z}\left(-1-c_{1} c_{2}+c_{3} c_{4}\right)=-c_{1} \delta \\ \beta\left(-c_{5}+c_{6}\right)+\omega_{z}\left(-c_{2} c_{5}+c_{4} c_{6}\right)=-c_{5} \delta\end{array}\right.$

The matrix is thus obtained:

$\mathbf{A}=\left[\begin{array}{cc}-c_{1}-c_{3} & -1-c_{1} c_{2}+c_{3} c_{4} \\ -c_{5}+c_{6} & -c_{2} c_{5}+c_{4} c_{6}\end{array}\right]$

The type of critical points (also called singular points, or equilibrium points) depends on the eigenvalues $\lambda_{1}$ and $\lambda_{2}$ of the matrix A; at the same time, the eigenvalues also show whether a critical point is stable or unstable [5]:

- if $\lambda_{1}$ and $\lambda_{2}$ are real, one positive and one negative, then there is a critical point of type saddle, which is an unstable critical point, an example being shown in fig. 5;

- if $\lambda_{1}$ and $\lambda_{2}$ are real and of the same sign, then there is a node-type critical point; if $\lambda_{1}$ and $\lambda_{2}$ are negative then there is a stable node, and if they are positive then there is an unstable node;

- if $\lambda_{1}$ and $\lambda_{2}$ are complex-conjugate, then there is a focal point critical point; if the real part of both eigenvalues is negative then there is a stable focus (with an example in fig. 6), and if it is positive then there is an unstable focus. 


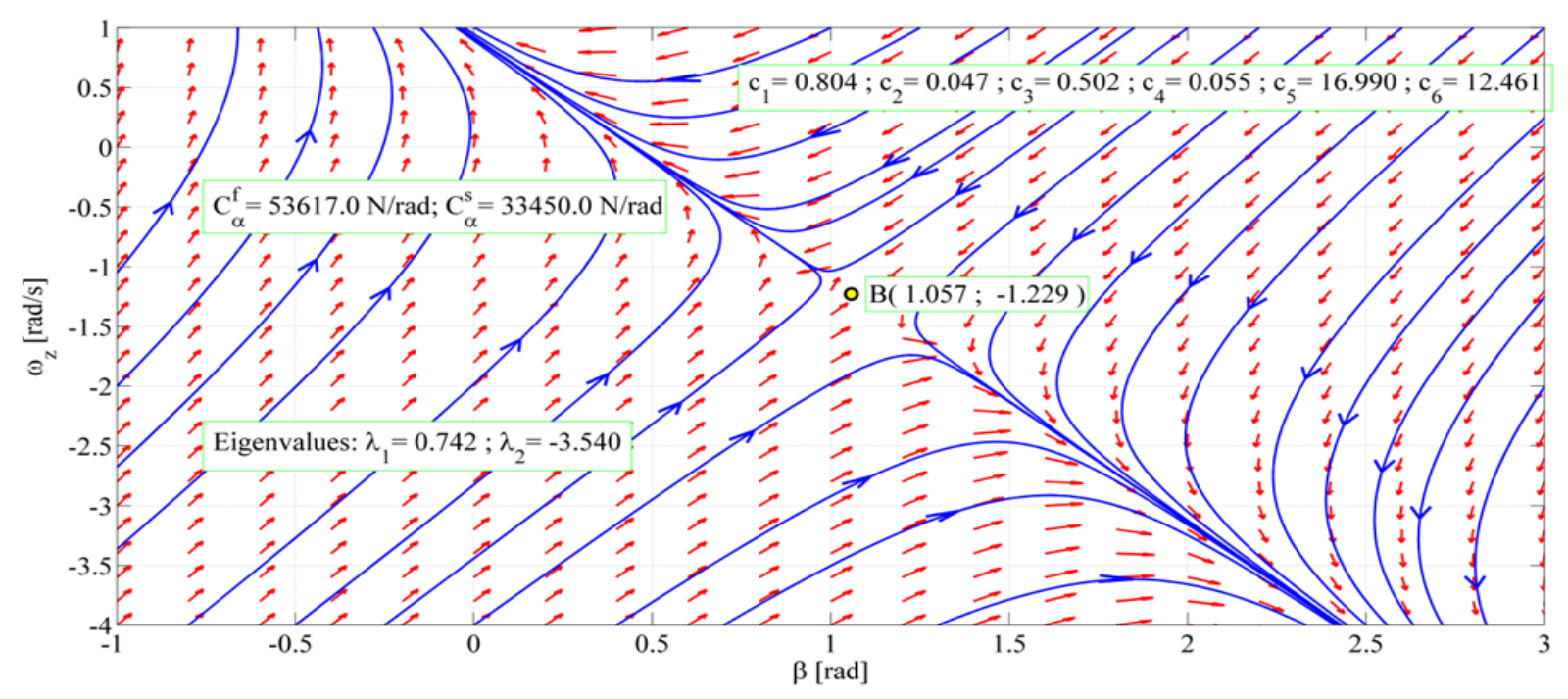

Figure 5 ESC stability study at different tire stiffness values

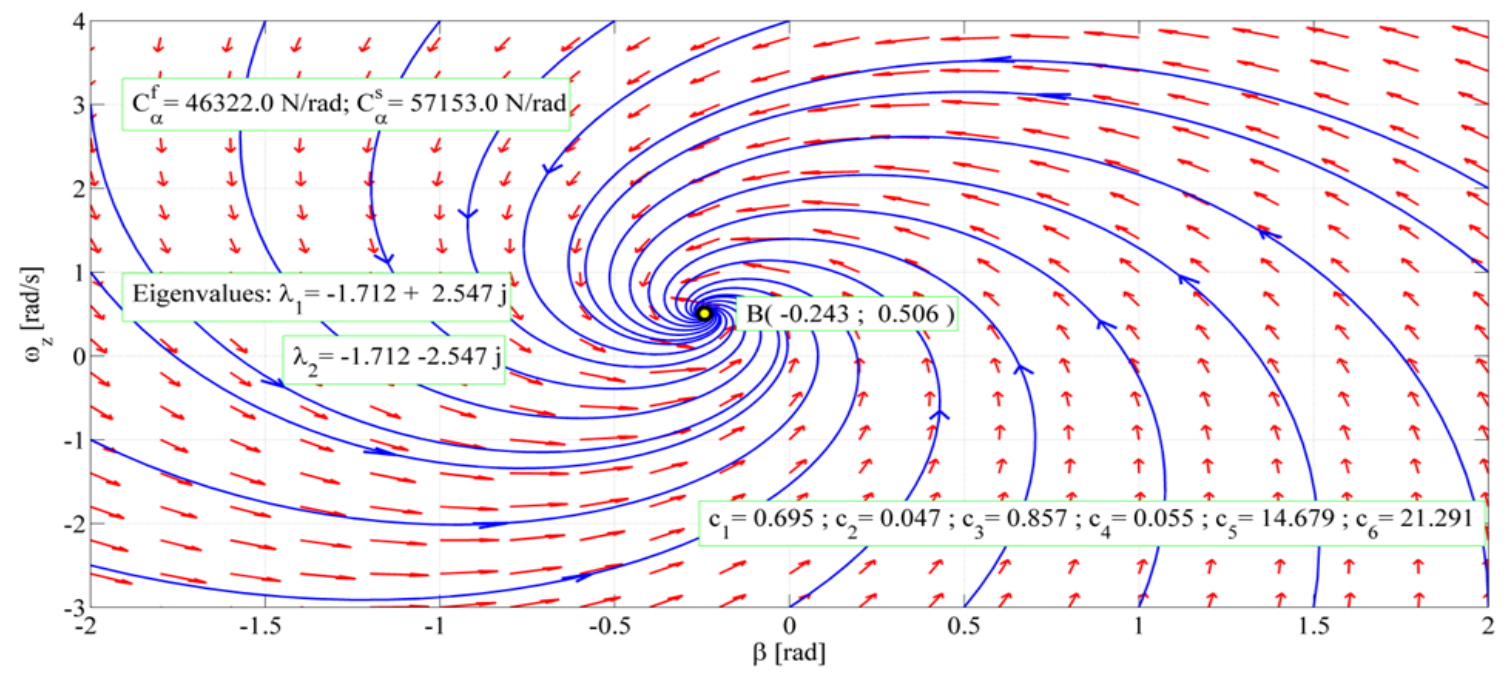

Figure 6 ESC stability study at different tire stiffness values

Fig. 5 and fig. 6 are shown the vector field, the phase trajectories, the values of the lateral stiffnesses of the tires, the eigenvalues of the matrix $\mathbf{A}$ and the values of the coefficients of the mathematical model; the calculations were performed by changing only the values of the lateral stiffness of the tires, to mark their importance in ensuring the stability of the lateral dynamics.

In this way other aspects can be addressed. For example, fig. 7a shows the determination of the maximum longitudinal speed that ensures the stability of the lateral dynamics for certain values of tire stiffness. As can be seen from fig.7a, the maximum longitudinal speed for stability reasons is less than $151.4 \mathrm{~km} / \mathrm{h}$, value over which one of the poles $p$ of the transfer function becomes positive; the poles of the transfer function are the roots of the characteristic equation (8). This aspect is confirmed in fig. $7 \mathrm{~b}$ for $V_{x}=180 \mathrm{~km} / \mathrm{h}$, at which the two quantities do not tend towards a finite value but towards infinity cumulative with time.

Similarly, the minimum values of the lateral stiffness of the tires can be set at a certain longitudinal speed so as to ensure the stability of the lateral dynamics of the car. 
a) Poles of transfer function

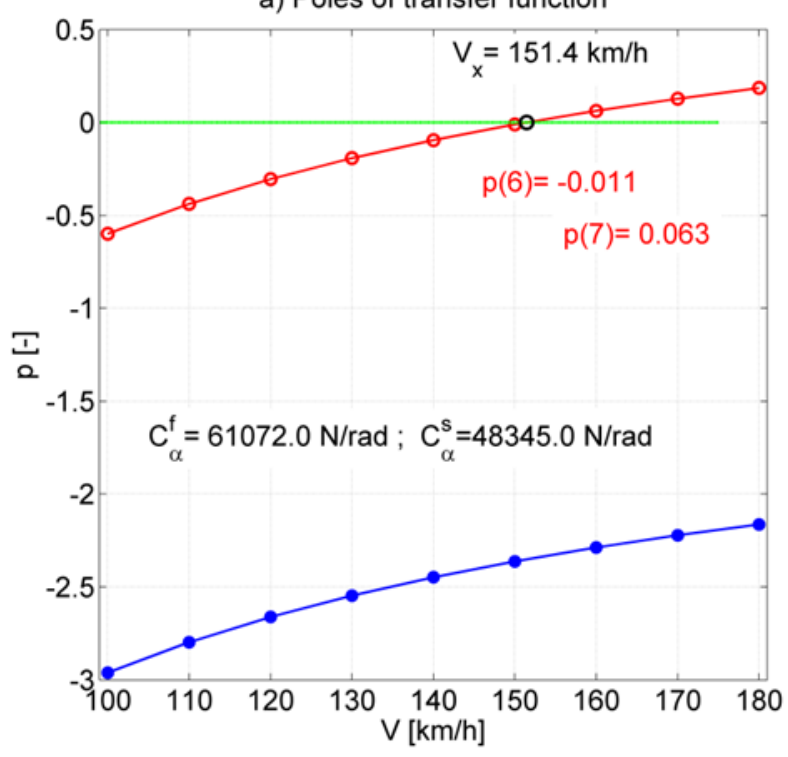

b) The speed of $180 \mathrm{~km} / \mathrm{h}$

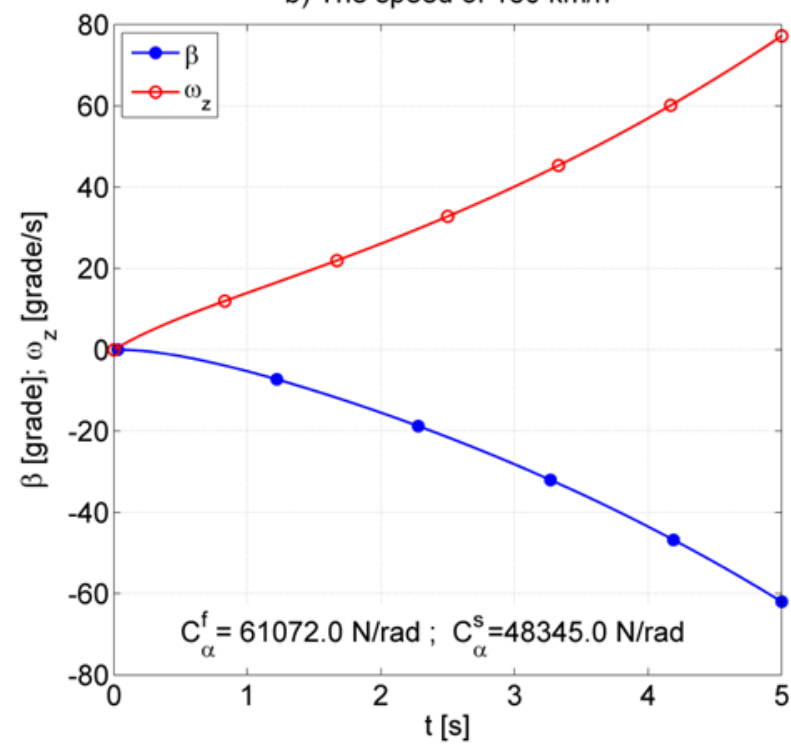

Figure 7 ESC stability study at different speeds

\subsection{Lateral dynamics control}

One of the important problems is the electronic control of the lateral dynamics of the car, the main role of the ESC system. There are three control strategies frequently applied to lateral dynamics control systems $[6,7,8]$ :

- differential braking, which uses the ABS braking system for the different application of braking to the car's wheels in order to control the torque;

- active steering control, which ensures the correction of the steering angle given by the driver, so that the vehicle returns to the desired/imposed trajectory;

- active distribution of torque to the wheels, which uses active differential and traction on all wheels, to control the traction torque.

In order for the ESC to fulfill its role, the vehicle is composed of transducers for measuring longitudinal acceleration, lateral acceleration, angular speed, wheel speed, steering angle and braking pressure.

For example, Fig. 8 shows the movement on a slalom route of a Chevrolet Camaro car that moved with ESC out of order and ESC functional, with the detail in fig. 9. As it turns out, in case of decommissioning of the ESC system, the car leaves the required route, has a pronounced turning motion, which results in loss of control by the driver, thus increasing the risk of a car accident.

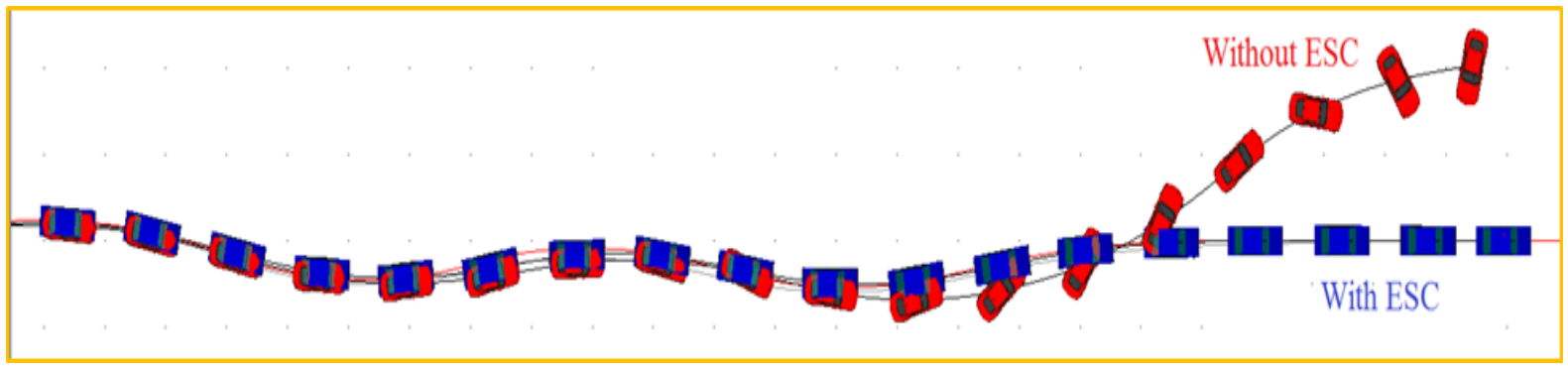

Figure 8 Driving on an imposed slalom course without ESC and with ESC 


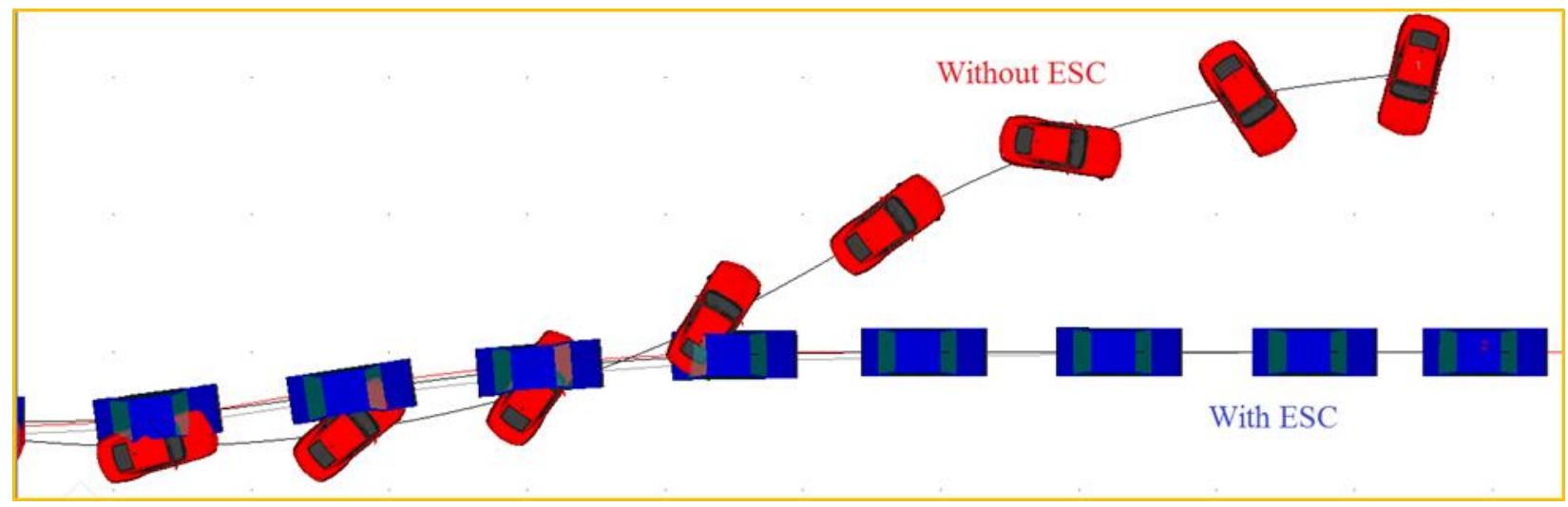

Figure 9 Driving on an imposed slalom course without ESC and with ESC, detail

Fig. 10 shows the parameteres related to the turning movement (upper graphs) and the steering angles of the front wheels (lower graphs).

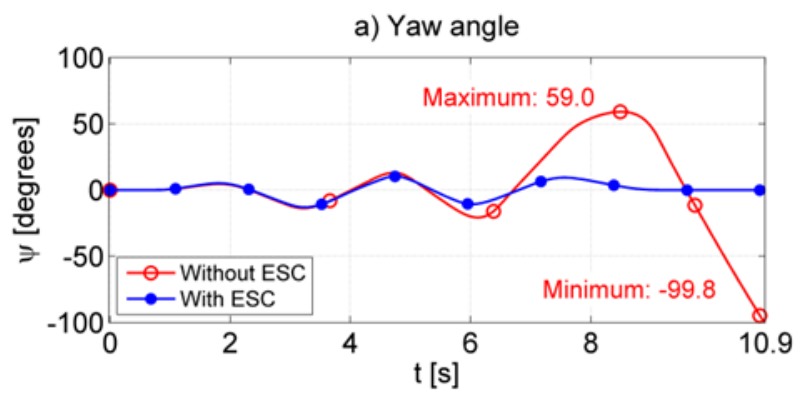

c) Wheel steering angles, without ESC

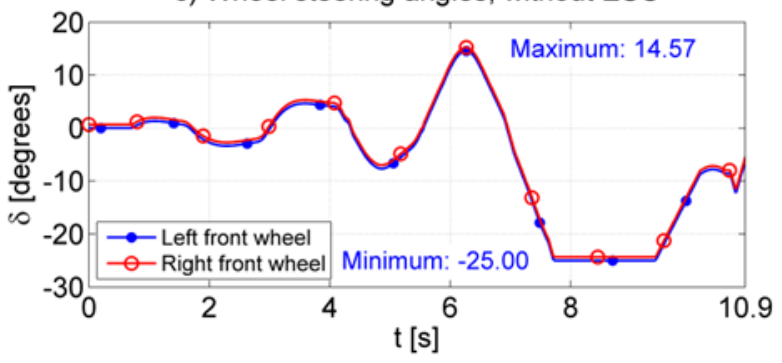

b) Angular velocity of the yaw motion (yaw rate)

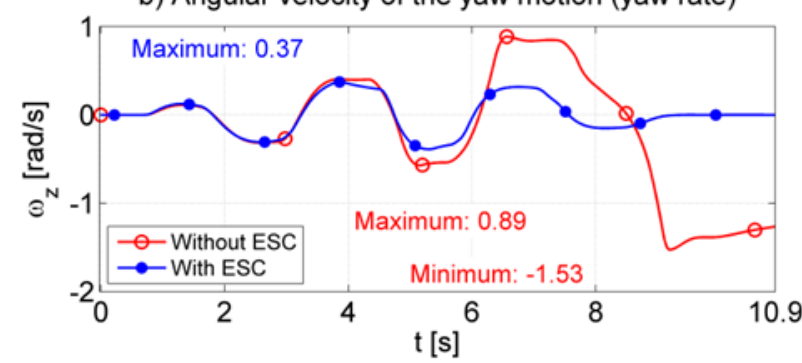

d) Wheel steering angles, with ESC

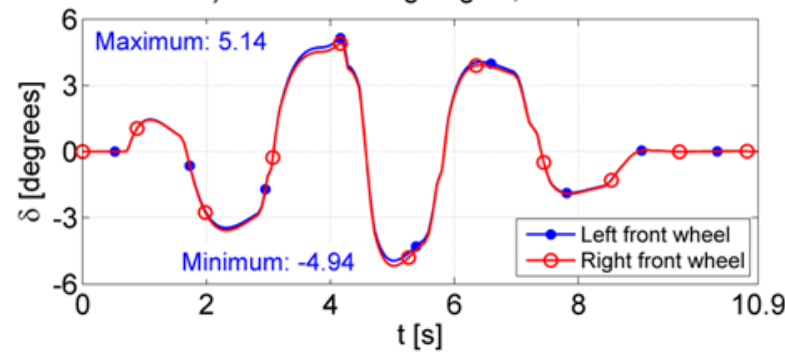

Figure 10 Yaw angle, yaw rate and wheel steering angles

As can be seen from the upper graphs in fig.10, in the case of ESC malfunction, the rotation movement has amplified over time. For example, the rotation angle increased to 99.8 degrees clockwise (it has a negative value), which can be seen in fig.9.

The lower graphs in fig.10 show the pronounced increase of the steering angles of the wheels in case of ESC malfunction.The graphs in fig.11 show the application of the differential braking algorithm of the wheels to keep the car on the required route in case of ESC operation. 
a) Left front wheel

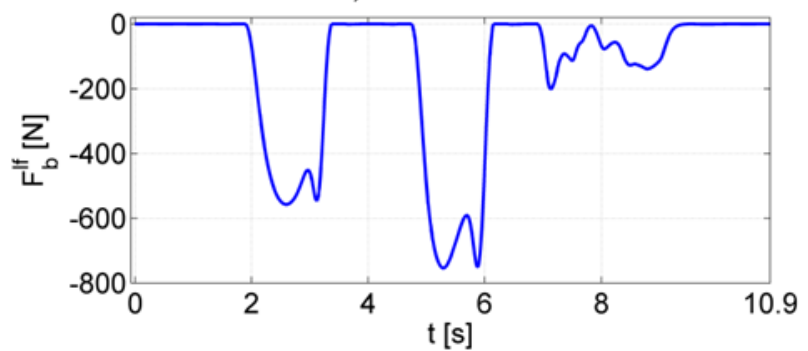

c) Left rear whee

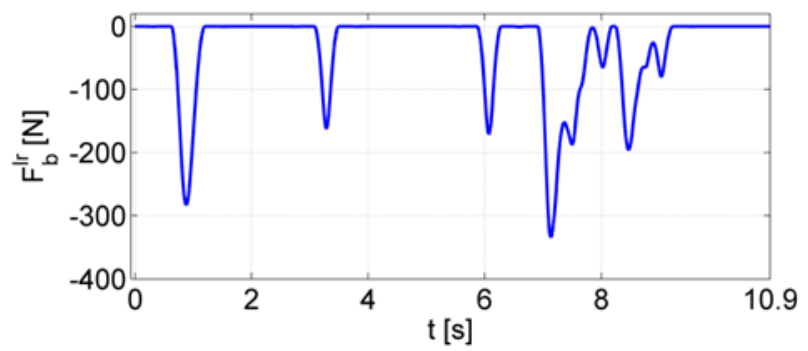

b) Right front wheel

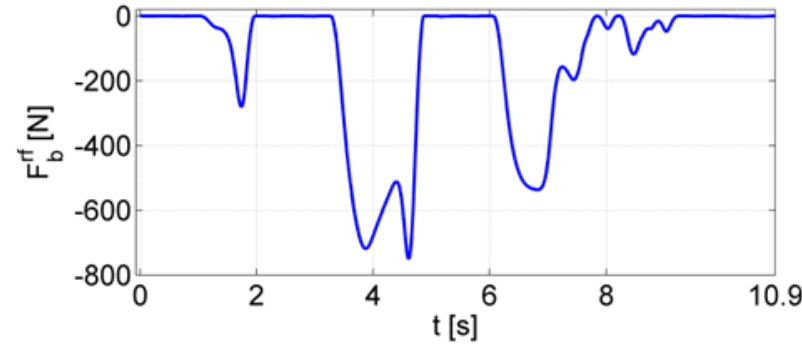

d) Right rear wheel

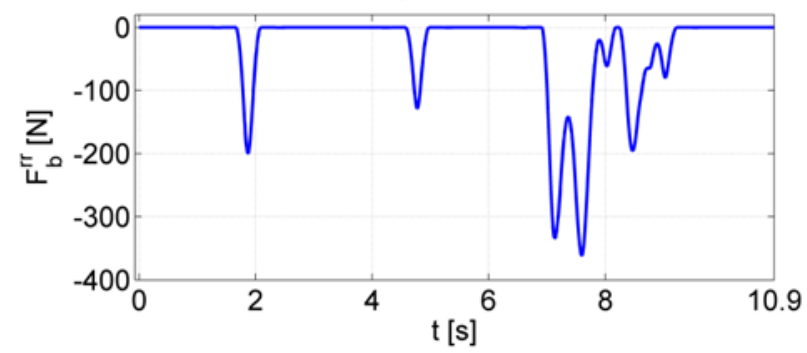

Figure 11 Wheel braking forces with ESC in operation, movement on wet asphalt

Differential wheel braking satisfies the control algorithm of the ESC system, which aims to obtain the smallest possible values of two quantities: the distance between the center of gravity of the car and the required trajectory, as well as the difference between the required turning angle and the actual turning angle.

ESC systems used on current vehicles use various control algorithms in order to obtain required performance, the main ones being: feedback loop control; PID control; adaptive control; MPC - Model Predictive Control; SMC - Sliding Mode Control; intelligent control (based on artificial intelligence algorithms: genetic algorithms, fuzzy logic, neural networks, neuro-fuzzy algorithms); robust control ( $\mathrm{H}_{2}$ control and $\mathrm{H} \infty \mathrm{control}$ ); FTC - Fault Tolerant Control.

\section{Conclusion}

Electronic control of the lateral dynamics is of great importance, because an impermissible deviation of the trajectory from the one desired by the driver can lead to road accidents with serious consequences for people and significant damage to the vehicles involved. For this reason, the control and stability of the lateral dynamics concern aspects related to the vehicle, the road and the driver.

For the study of lateral motion stability, algorithms from systems theory are applied, the phase portrait being widely used, which allows the establishment and visualization of critical points.

The presented study highlights the need to establish the speed and lateral stiffness of tires that lose the stability of lateral movement.

For a more in-depth study of lateral dynamics, other mathematical models can be used, with an increased number of degrees of freedom $[9,10,11]$.

\section{References}

[1] Aripin M. A review of active yaw control system for vehicle handling and stability. IJVT. 2014; 43: 1-15.

[2] Cao J. Integrated control of vehicle yaw and rollover stability. Jiao Tong University. 2013.

[3] Naser I. H. A review of speed - flow relationships in traffic studies. Global Journal of Engineering and Technology Advances (GJETA). 2021; 06(01): 026-035.

[4] Andreasson J. On generic road vehicle motion modelling and control. Thesis, Royal Institute of Technology. Stockolm. 2007. 
[5] Bobier C. A phase portrait approach to vehicle stabilization and envelope control. PhD thesis. Stanford University. 2012.

[6] Guoye W. Study of ESP control. International Conference of SSDMS. 2012.

[7] Stoica RM, Radulescu VJ, Neagu D, Trocan C, Copae I. Aspects Regarding the Analysis and Reconstruction of Car Crashes. ROJAE. 2017; 23: 113 .

[8] Zhao L. The optimization research on vehicle ESP control strategy. Applied Mechanics and materials. 2013; 321324: 1548-1553.

[9] Berntorp K. Derivation of a six degrees of freedom ground vehicle model for automotive applications. Thesis. Lund University. 2013.

[10] Pantil V. Generic and complete vehicle dynamic models for open-source platform. Chalmers University of Technology, Gothenburg. 2017.

[11] Sokolovskij E. Modelling of collisions of the automobiles. Technical University. Vilnius. 2006. 